\title{
ON THE COMPUTATIONAL FLUID DYNAMICS SIMULATIONS OF THE SHIP NOMINAL WAKE IN THE PROPELLER PLANE FOR FULL SHIPS
}

\author{
AleKsandrina VASILEVA \\ Institute of Metal Science, Equipment and Technologies \\ with Hydro- and Aerodynamics Centre "Acad. Angel Balevski", \\ Bulgarian Academy of Sciences \\ 1, William Froude St., 9003 Varna, Bulgaria, \\ e-mail: a.vasileva@bshc.bg
}

\begin{abstract}
The main objective of this paper is to investigate the applicability of Computational Fluid Dynamics (CFD) to estimate the nominal wake in the propeller plane for single screw $300 \mathrm{k}$ DWT VLCC. The investigated model is tested in two cases - Bare hull case and Ship fitted with energy saving device. All CFD calculations are compared with results of model tests. Four turbulent models were tested to assess whether the specific features of the flow around full ships with conventional sterns are well captured. The validation with model tests data proved that the Reynolds stress transport model performed best.

Keywords: Computational fluid dynamics, turbulence, nominal wake.
\end{abstract}

\section{INTRODUCTION}

The nominal wake field is the wake field that would be measured at the propeller plane without the presence or influence of the propeller modifying the flow at the stern of the ship [1]. That nominal wake distribution is of particular importance for propeller designers because it shows some specific features of ship flow at the stern.

It can be seen in Fig. 1 that the wake pattern features a rotation of the flow into the propeller. This type of rotation is called a bilge vortex. This vortex creates an exchange of water: low-speed water particles from boundary layer are moved away from the hull, while high-speed water particles outside the boundary layer are transported into the close vicinity of the hull surface. Obviously, the detailed shape of the velocity contours has an important effect on the operation of the propeller.

DOI: 10.7546/EngSci.LVIII.21.02.04 
The ship design tend is to improve the flow in order to move the contours out of the central and lower parts of the propeller disk and to squeeze them at the top of the disk. To find the optimum solution, the position of the vortex is important from a hull efficiency and vibration point of view, and may call for considerable experimentation model testing and prediction with Computational Fluid Dynamics (CFD) [2]. Adequate numerical simulations can save significant time and resources for model tests.

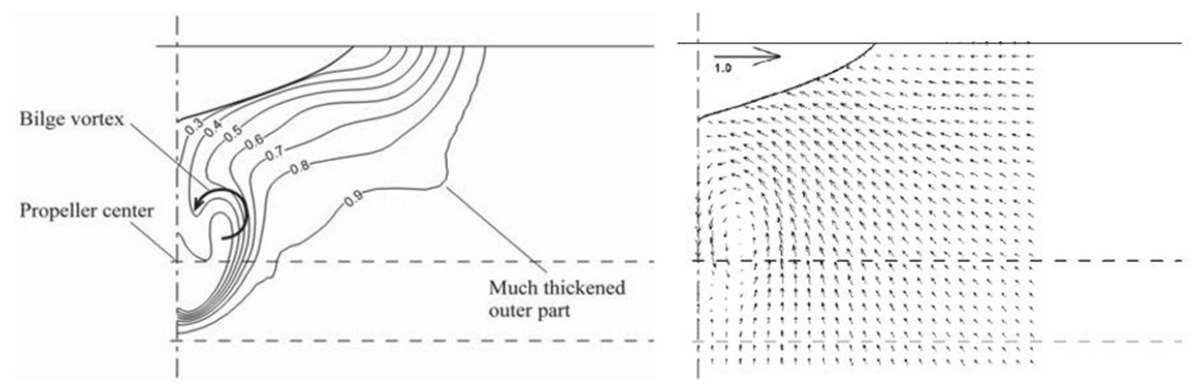

Fig. 1. Contour of constant axial velocity and projection of velocity vectors at the propeller plane [2]

\section{CFD METHODOLOGY}

The software used for CFD calculations is academic package of STAR $\mathrm{CCM}+$. For the model fluid flow the current CFD simulations impose finite volume methods, which use the integral form of the conservation equations and divide the computational domain into finite number of joined control volumes. The transient turbulent multiphase simulation is set.

The present setting considers a viscous and incompressible fluid, turbulent flow and solves the Reynolds-Averaged Navier-Stokes Equations (RANSEs). The RANSEs determine the average values of the physical variables (velocity and pressure).

To account for the influence of turbulence, turbulent models are constructed and used: these are calculation procedures of closing the system of equations for the averaged flow.

There are two basic approaches for RANS turbulence models used in STAR CCM+ [3]: (i) Eddy viscosity models, and (ii) Reynolds stress transport models. The applicability of each of models is shown below.

Engineering Sciences, LVIII, 2021, No. 2 


\subsection{Eddy viscosity models include three major classes of turbulent models}

- Spalart-Allmaras models are a good choice for applications, in which the boundary layers are largely attached, and separation is mild if it occurs. Typical examples would be the flow over a wing, the fuselage or other aerospace external-flow applications.

- K-epsilon models provide a good compromise between robustness, computational cost and accuracy. They are generally well suited to industrial-type applications that contain complex recirculation, with or without heat transfer.

The V2F K-epsilon model, a version of K-epsilon model, is used for calculations. It is designed to handle wall effects in turbulent boundary layers and to accommodate non-local effects. It is known to capture the near-wall turbulence effects more accurately, which is crucial for the accurate prediction of heat transfer, skin friction and flow separation.

- K-omega models are similar to K-epsilon models in that two transport equations are solved, but differ in the choice of the second transported turbulence variable. The performance differences are likely to be a result of the subtle differences in the models, rather than a higher degree of complexity in the physics being captured. Therefore, they are recommended as an alternative to the Spalart-Allmaras models for similar types of applications.

The SST (Menter) K-omega Detached Eddy model, a version of K-omega model, is used for calculations. It allows to combine features of the SST (Menter) K-omega RANS model in the boundary layers with a Large Eddy Simulation (LES) in unsteady separated regions.

\subsection{Reynolds stress transport models}

Reynolds stress transport models are the most complex and computationally expensive models offered in STAR-CCM+. They are recommended for situations in which the turbulence is strongly anisotropic, such as the swirling flow in a cyclone separator.

STAR-CCM+ has a choice of four different Reynolds stress transport turbulence models:

- Linear Pressure Strain Model;

- Quadratic Pressure Strain Model;

- Linear Pressure Strain Two-Layer Model;

- Elliptic Blending Model. 
The Quadratic Pressure Strain model and its low-Reynolds number variant, the Elliptic Blending model are based on the most recent and precise formulation of the pressure-strain term. Thus, they are likely to be the models of choice in a majority of cases, the Elliptic Blending model being preferred as it also contains specific treatments for all-Y+ meshes. However, the Linear Pressure Strain model is also available with a two-layer formulation, thus also applicable down to the wall.

For the current investigations the main four types of turbulent models are used: Spalart-Allmaras models, K-epsilon model (V2F), K-omega model (SST Menter) and Reynolds stress transport model (Elliptic Blending model).

\section{TEST CASE}

All further discussions in this paper are based on observations of EFD and CFD calculations for a 300k DWT VLCC with main particulars for design loading condition given in Table 1.

Table 1. Principle dimensions

\begin{tabular}{|l|c|c|c|l|}
\hline Particulars & \multicolumn{2}{|c|}{ Symbol/Dim } & Ship & Model \\
\hline Length between perpendiculars & $\mathrm{L}_{P P}$ & {$[\mathrm{~m}]$} & 321.90 & 7.5210 \\
\hline Beam WL & $\mathrm{B}_{W L}$ & {$[\mathrm{~m}]$} & 60.00 & 1.4019 \\
\hline Draught & $\mathrm{T}$ & {$[\mathrm{m}]$} & 20.50 & 0.4790 \\
\hline Block coefficient & $\mathrm{C}_{B}$ & {$[-]$} & 0.797 & 0.7970 \\
\hline Velocity & $\mathrm{V}$ & {$[\mathrm{kn}],[\mathrm{m} / \mathrm{s}]$} & 14.800 & 1.1637 \\
\hline Propeller Diameter & $\mathrm{D}_{P}$ & {$[\mathrm{~m}]$} & 10.700 & 0.2500 \\
\hline Direction of rotation & & & \multicolumn{3}{|c|}{ clockwise } \\
\hline
\end{tabular}

CFD calculations are done for two cases: 300k DWT VLCC - Bare Hull shown in Fig. 1, and 300k DWT VLCC - fitted with Energy Saving Device (ESD) shown in Figs 2, 3 and 4. The simulations are carried out in model scale. The grid size for cases is given in Table 2 .

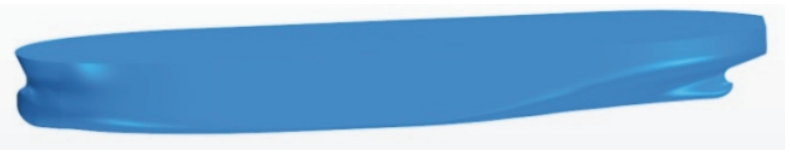

Fig. 2. Bare hull 


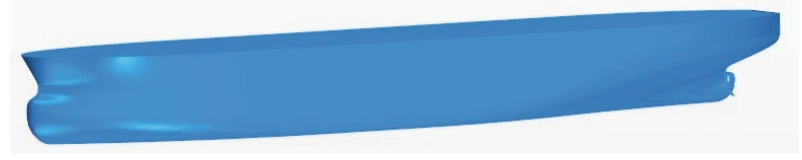

Fig. 3. Hull with ESD

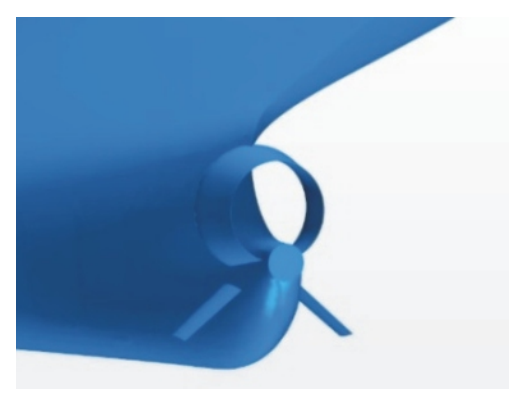

Fig. 4. Hull with ESD (Stern close-up)

Table 2. Grid size

\begin{tabular}{|l|c|}
\hline \multicolumn{1}{|c|}{ Case ID } & Grid size \\
\hline Bare Hull (using symmetry) & 3562497 \\
\hline Hull mounted with ESD & 5997483 \\
\hline
\end{tabular}

\section{RESULTS AND ANALYSIS OF CFD CALCULATIONS AND MODEL TESTS}

All results from physical model tests (EFD) and CFD calculations are presented in the same way for both cases (Bare Hull and Hull fitted with ESD) - figures of iso-curves of axial velocities and transverse velocity vectors, Figs

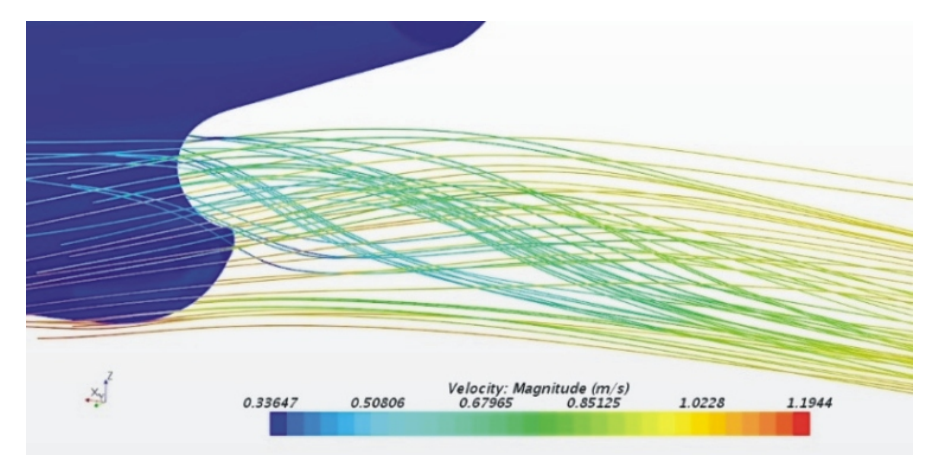

Fig. 5. Streamlines at stern for Bare Hull 
6 and 13, and graphics of circumferential distribution of axial velocities for non-dimensional radii, Figs 7-11 and 14-18:

$$
r / R=0.4,0.6,0.8,1.0 \text { and 1.2. }
$$

CFD calculations are presented for all investigated turbulent models: SpalartAllmaras models, K-epsilon model (V2F), K-omega model (SST Menter) and Reynolds stress transport model (Elliptic Blending model). Pictures of streamlines at stern are also shown, Figs 5 and 12.
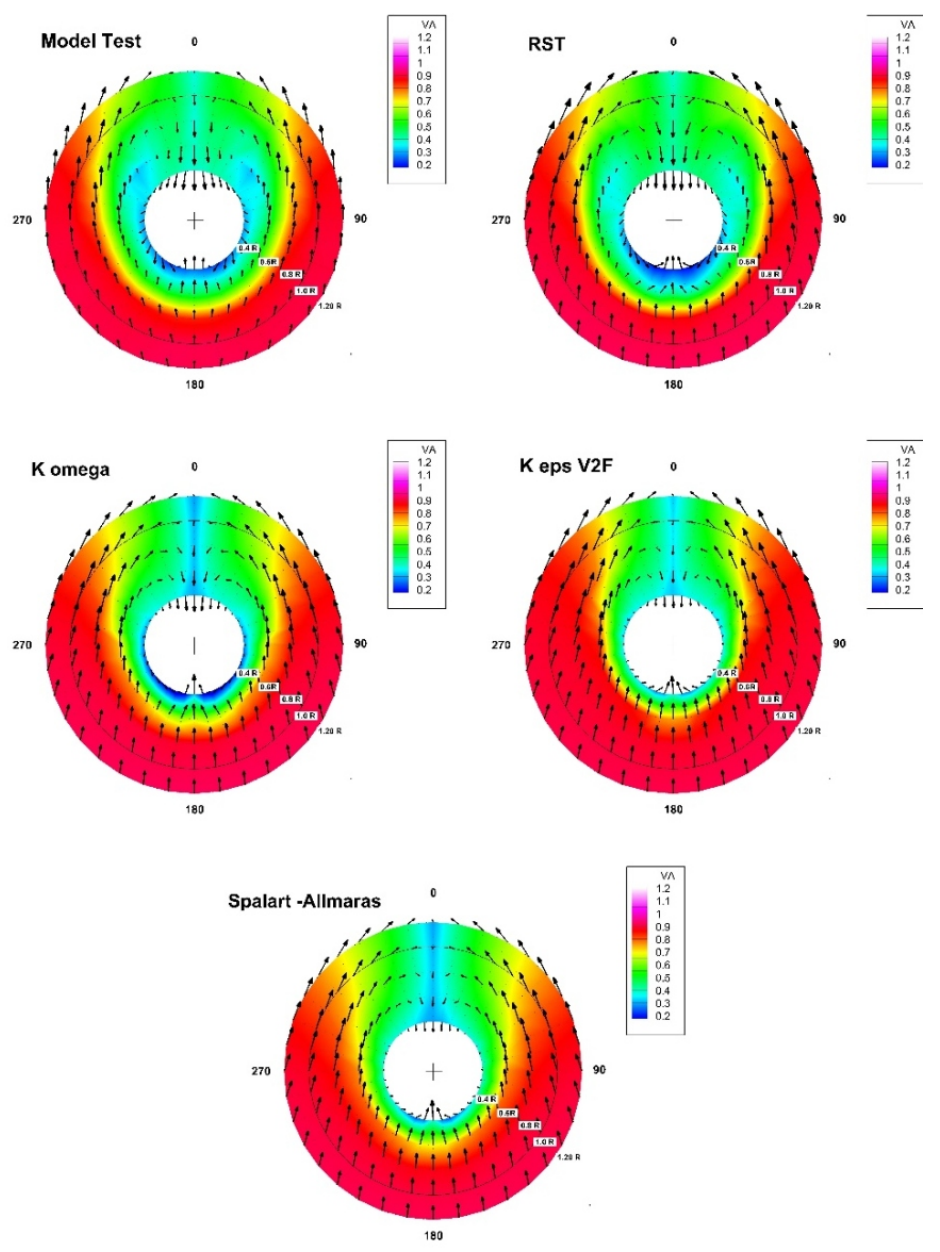

Fig. 6. Iso-curves of axial velocities and transverse velocity vectors for all Bare Hull tested turbulent models and model test 


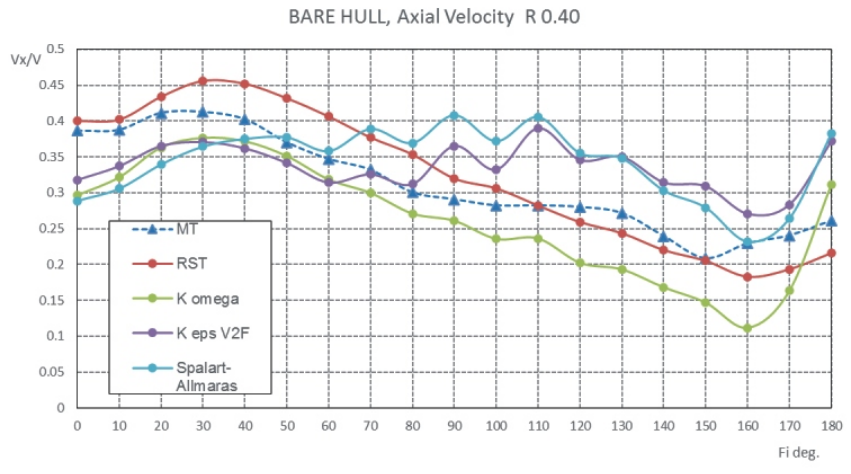

Fig. 7. Circumferential distribution of axial velocities for Bare Hull, $r / R=0.4$

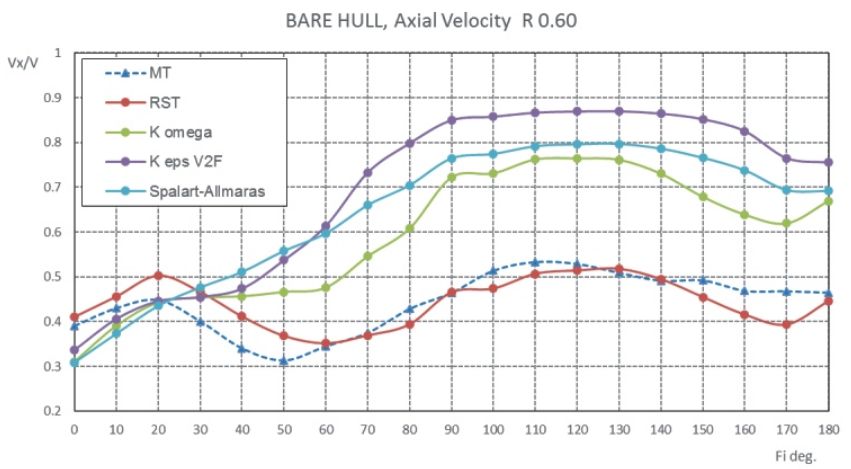

Fig. 8. Circumferential distribution of axial velocities for Bare Hull, $r / R=0.6$

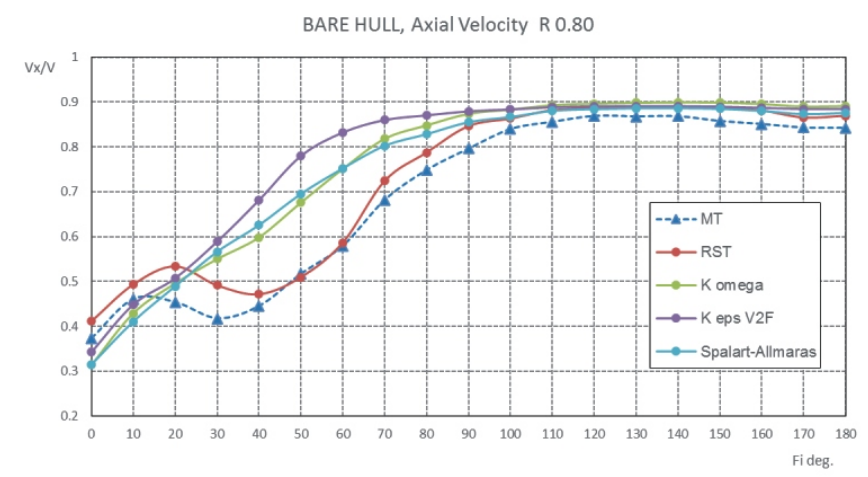

Fig. 9. Circumferential distribution of axial velocities for Bare Hull, $r / R=0.8$ 
BARE HULL, Axial Velocity R 1.00

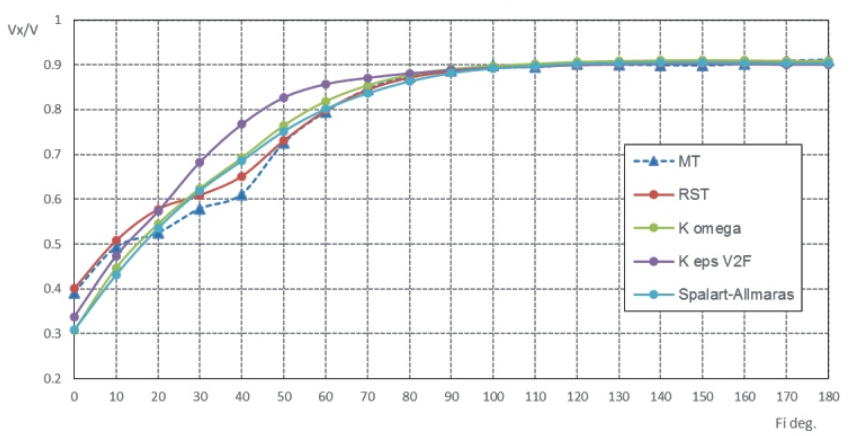

Fig. 10. Circumferential distribution of axial velocities for Bare Hull, $r / R=1.0$

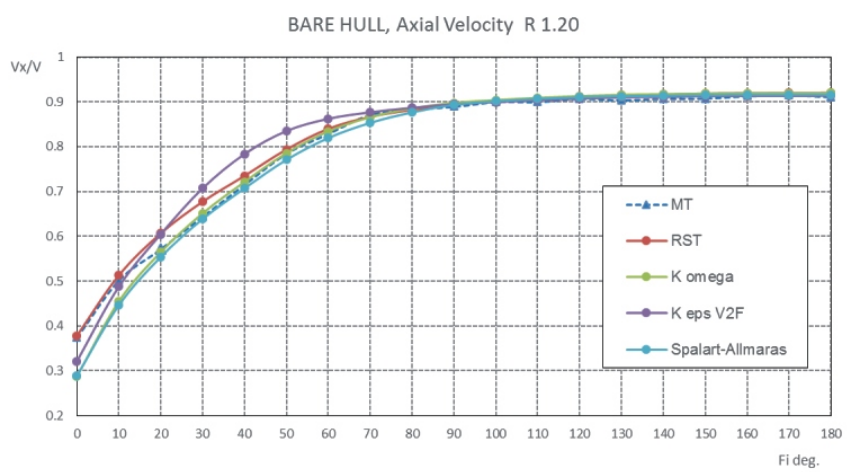

Fig. 11. Circumferential distribution of axial velocities for Bare Hull, $r / R=1.2$

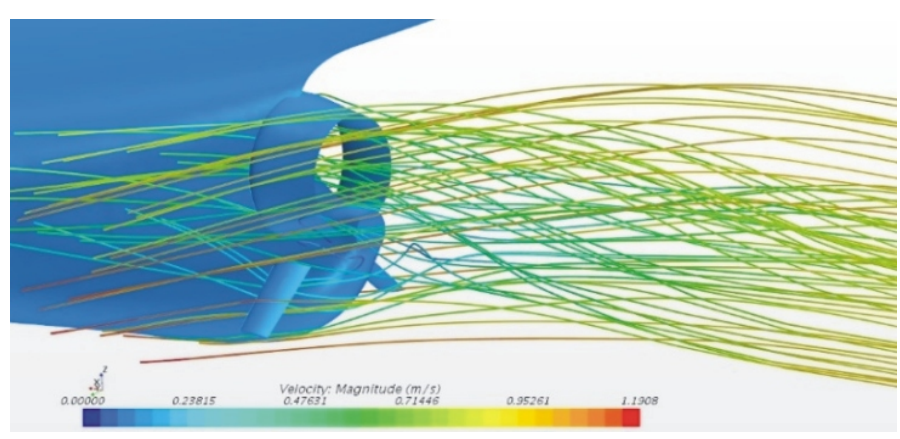

Fig. 12. Streamlines at stern for Hull with ESD 

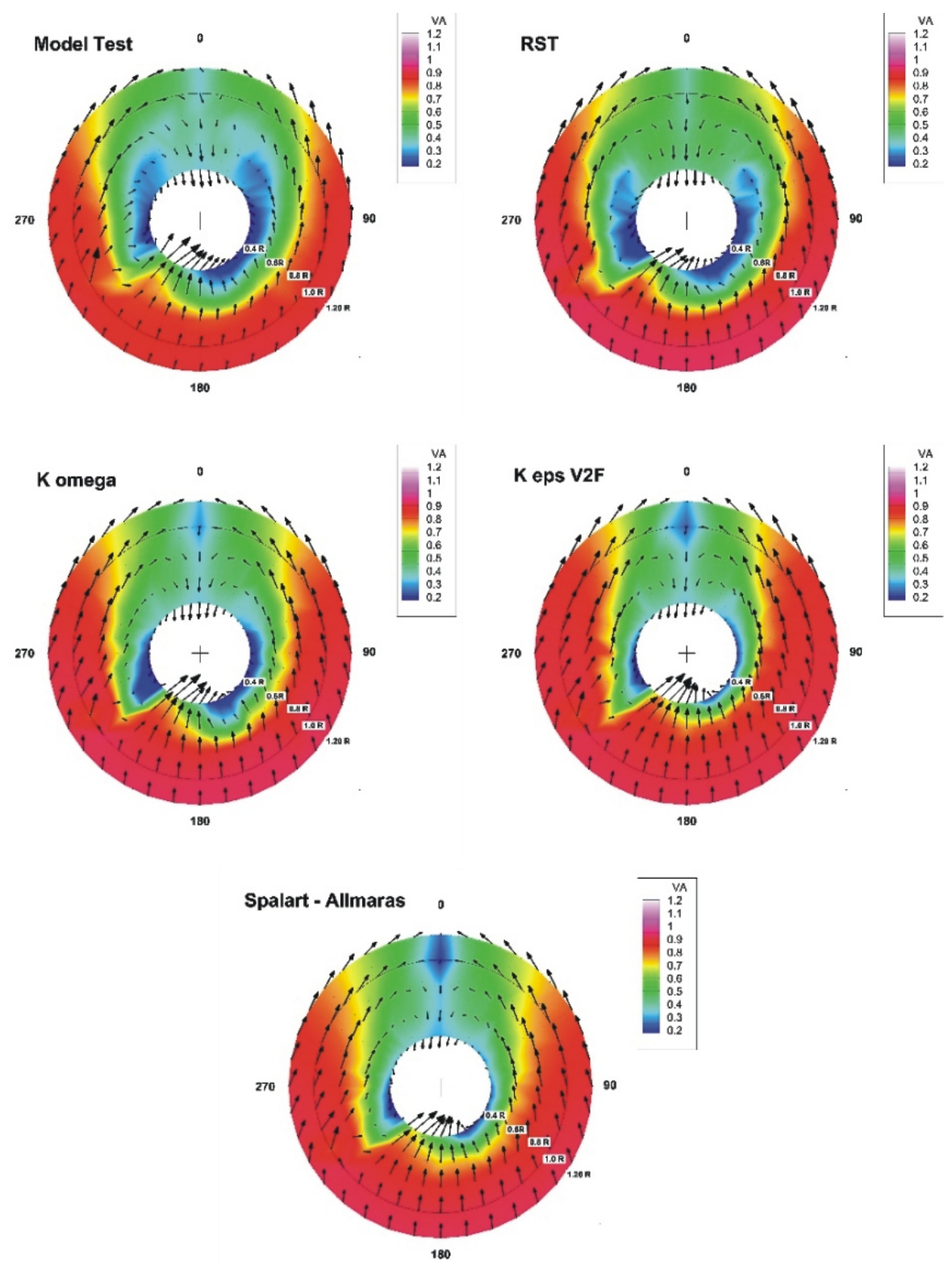

Fig. 13. Iso-curves of axial velocities and transverse velocity vectors for all Hull with ESD tested turbulent models and model test 
Hull with ESD, Axial Velocity R 0.40

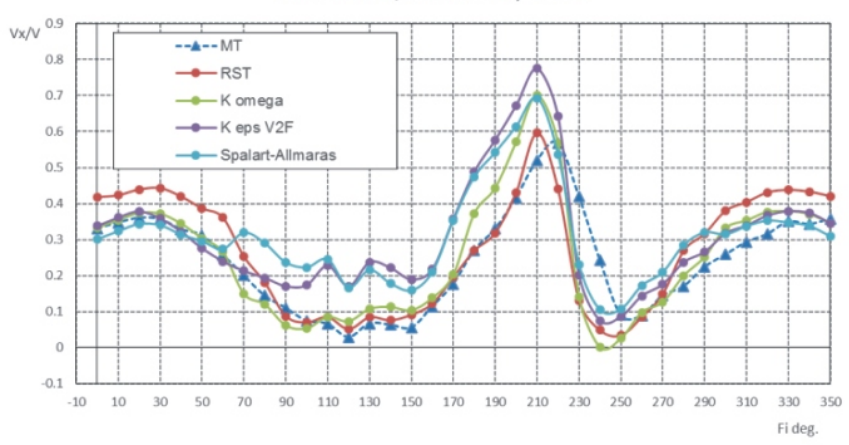

Fig. 14. Circumferential distribution of axial velocities for Hull with $\mathrm{ESD}, r / R=0.4$

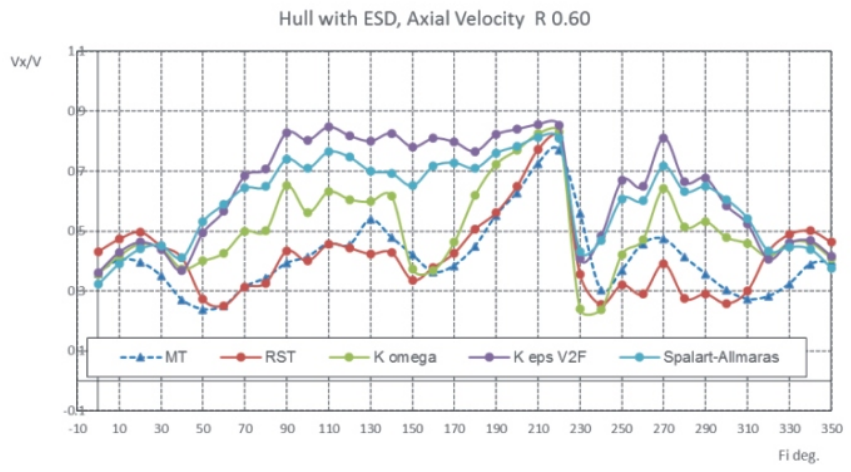

Fig. 15. Circumferential distribution of axial velocities for Hull with ESD, $r / R=0.6$

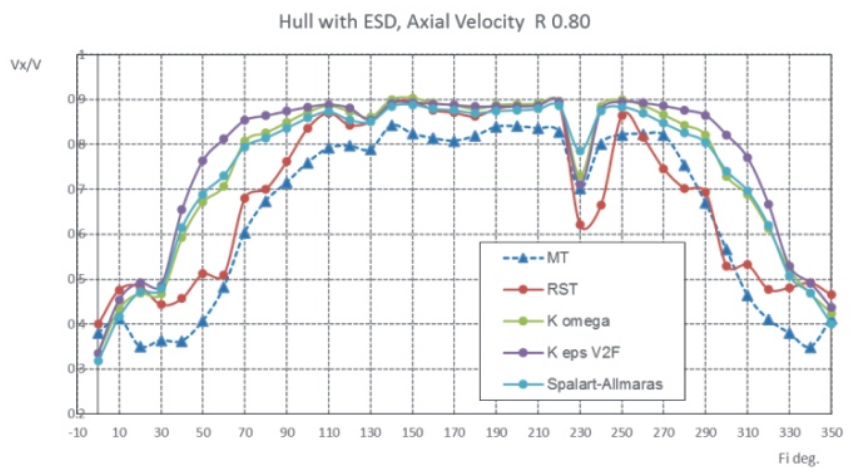

Fig. 16. Circumferential distribution of axial velocities for Hull with $\mathrm{ESD}, r / R=0.8$ 


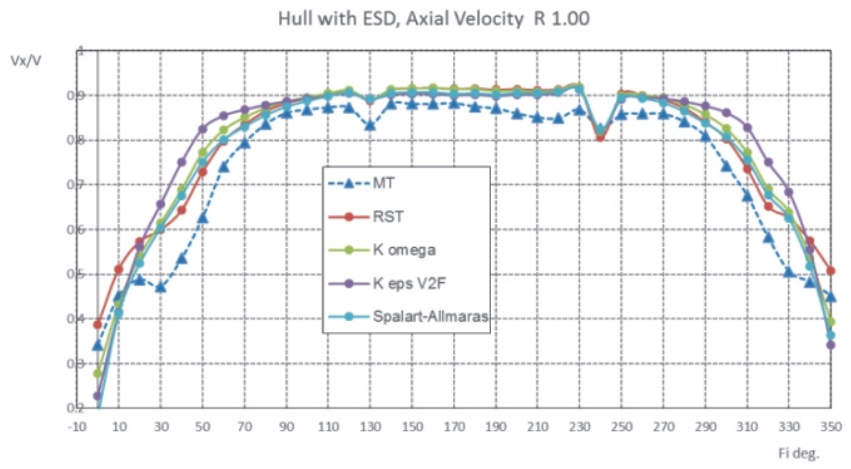

Fig. 17. Circumferential distribution of axial velocities for Hull with ESD, $r / R=1.0$

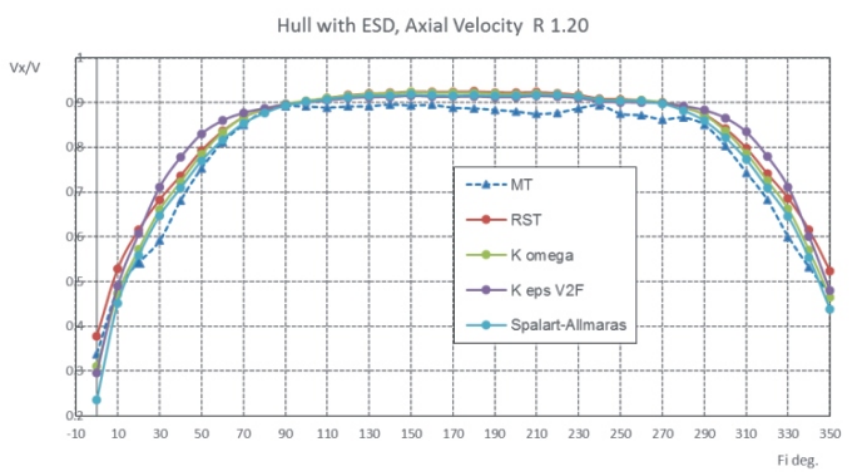

Fig. 18. Circumferential distribution of axial velocities for Hull with $\mathrm{ESD}, r / R=1.2$

\section{CONCLUSION}

On the basis of the results from CFD calculations for both cases - Bare Hull and Hull with ESD, it can be concluded that the Reynolds Stress Transport models describe most adequately the wake pattern for this type of ships.

The effect of the ESD is also satisfactorily predicted, which is encouraging with respect to the possibility of using CFD for optimum design of such devices.

\section{ACKNOWLEDGEMENTS}

Project BG05M2OP001-1.001-0008 National Center of Mechatronics and Clean Technologies. 


\section{REFERENCES}

[1] J. S. Carlton, Marine Propellers and Propulsion, Third Edition (2012).

[2] L. Larsson and H. Raven, Ship Resistance and Flow, The Principles of Naval Architecture Series (2010).

[3] STAR CCM+11, User Guide, Siemens, www.siemens.com.

[4] Lars Larsson and Frederick Stern, Numerical Ship Hydrodynamics, An Aassessment of the Gotheburg Workshop, Springer, 2010.

Received April 05, 2021

Engineering Sciences, LVIII, 2021, No. 2 